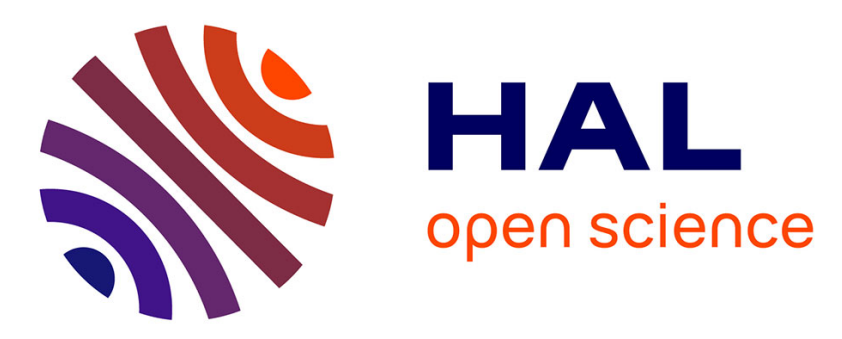

\title{
Linear stability of the 1D Saint-Venant equations and drag parameterizations
}

Olivier Thual, Louis-Romain Plumerault, Dominique Astruc

\section{To cite this version:}

Olivier Thual, Louis-Romain Plumerault, Dominique Astruc. Linear stability of the 1D Saint-Venant equations and drag parameterizations. Journal of Hydraulic Research, 2010, vol. 48, pp. 348-353. 10.1080/00221686.2010.481837 . hal-00951007

\section{HAL Id: hal-00951007 https://hal.science/hal-00951007}

Submitted on 24 Feb 2014

HAL is a multi-disciplinary open access archive for the deposit and dissemination of scientific research documents, whether they are published or not. The documents may come from teaching and research institutions in France or abroad, or from public or private research centers.
L'archive ouverte pluridisciplinaire HAL, est destinée au dépôt et à la diffusion de documents scientifiques de niveau recherche, publiés ou non, émanant des établissements d'enseignement et de recherche français ou étrangers, des laboratoires publics ou privés. 


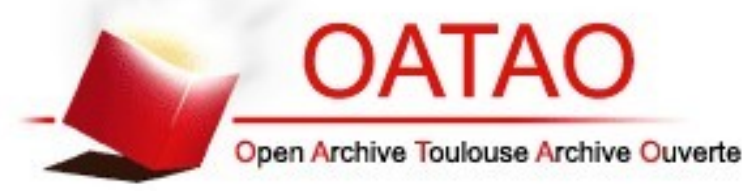

\section{Open Archive TOULOUSE Archive Ouverte (OATAO)}

OATAO is an open access repository that collects the work of Toulouse researchers and makes it freely available over the web where possible.

This is an author-deposited version published in : http://oatao.univ-toulouse.fr/ Eprints ID : 6707

To link to this article : DOI:10.1080/00221686.2010.481837

URL : http://dx.doi.org/10.1080/00221686.2010.481837

To cite this version : Thual, Olivier and Plumerault, Louis-Romain and Astruc, Dominique Linear stability of the 1D Saint-Venant equations and drag parameterizations. (2010) Journal of Hydraulic Research , vol. 48 (n 3 ). pp. 348-353. ISSN 0022-1686

Any correspondance concerning this service should be sent to the repository administrator: staff-oatao@,listes-diff.inp-toulouse.fr 


\title{
Linear stability of the 1D Saint-Venant equations and drag parameterizations
}

\section{Stabilité linéaire des équations de Saint-Venant 1D et paramétrisations du frottement}

Olivier THUAL, Université de Toulouse; INPT, UPS; IMFT, Allée Camille Soula, F-31400 Toulouse, France.CNRS; IMFT; F-31400Toulouse,France.E-mail: thual@imft.fr

Louis-Romain PLUMERAULT, Université de Pau et des Pays le l'Adour; LAGASEC2, 1, allée du Parc Montaury, F-64600 Anglet, France.E-mail: louisromain@yahoo.fr

Dominique ASTRUC, Université de Toulouse; INPT, UPS; IMFT, Allée Camille Soula, F31400 Toulouse, France.CNRS; IMFT; F-31400Toulouse,France.E-mail: astruc@imft.fr

\begin{abstract}
The stability of the homogeneous and steady flow based on the one-dimensional Saint-Venant equations for free surface and shallow water flows of constant slope is derived and displayed through graphs. With a suitable choice of units, the small and large drag limits, respectively, correspond to the small and large spatio-temporal scales of a linear system only controlled by the Froude number and two other dimensionless numbers associated with the bottom drag parameterization. Between the small drag limit, with the two families of marginal and nondispersive shallow water waves, and the large drag limit, with the marginal and nondispersive waves of the kinematic wave approximation, dispersive roll waves are detailed. These waves are damped or amplified, depending on the value of the three control parameters. The spatial generalized dispersion relations are also derived indicating that the roll-wave instability is of the convective type for all drag parameterizations.
\end{abstract}

\section{Résumé}

La stabilité de l'écoulement homogène et stationnaire fondé sur les équations de Saint-Venant unidimensionelles pour des écoulements à surface libre en eaux peu profondes avec pente constante est calculée et représentée à l'aide de graphes. Avec un choix d'unités approprié, les limites des grands et petits frottements correspondent respectivement aux grandes et petites 
échelles spatiotemporelles d'un système linéaire seulement contrôlé par le nombre de Froude et deux autres nombres sans dimension associés au choix de la paramétrisation du frottement au fond. Entre la limite des faibles frottements, avec les deux familles d'ondes marginales et non dispersives en eaux peu profondes, et la limite des grands frottements, avec les ondes marginales et non dispersives de l'approximation de l'onde cinématique, les ondes dispersives « roll waves » sont décrites. Ces roll waves sont amorties ou amplifiées selon la valeur des trois paramètres de contrôle. Les relations de dispersion généralisées spatiales sont aussi dérivées indiquant que l'instabilité des ondes de rouleaux est de type convectif pour toutes les paramétrisations du frottement.

Keywords: Convective instability, kinematic-wave approximation, roll-wave instability, SaintVenant equations, shallow water

\section{Introduction}

The Saint-Venant equations, also referred to as shallow-water equations, constitute a model for free surface flows in which the horizontal scales are significantly larger than the flow depth. In typical hydraulic textbooks, including Chow (1959) or Akan (2006), these equations are derived from the incompressible Navier-Stokes equations. In this process, a wide range of bottom friction parameterizations are used, ranging from constant Chézy coefficients to the empirical Colebrook-White (1937) formula.

The linear stability problem of the steady solution of the one-dimensional (1D) SaintVenant equations, referred as normal flow, is well-known. If the bottom friction forces and the channel slope are neglected, small perturbations of the normal flow are classified in two families of both non-dispersive and un-damped waves. Their phase velocities are $U \pm(g h)^{1 / 2}$ where $U=$ fluid velocity, $h=$ flow depth and $g=$ gravity constant. If the bottom friction and the gravity forces dominate the inertia and the pressure forces, one finds a single family of non-dispersive and un-damped waves. Their phase velocity is $5 U / 3$ for the Strickler parameterization (e.g. Chow 1959). Between these two limit cases, if all the above forces are of the same order, amplified or damped dispersive waves characterize the linear dynamics of a small perturbation of normal flow.

The pioneering work of Jeffreys (1925) exhibited an instability for a Froude number $F=$ $U /(g h \cos \alpha)^{1 / 2}>2$ for constant Chézy parameterization, where $\alpha=$ bottom angle with the horizontal plane. This instability was used to explain 'roll waves' observed by Cornish 
(1910). Several works followed to study the nonlinear wave dynamics for laminar flow (Jeffreys 1925, Kapitza and Kapitza 1948, Benjamin 1957, Yih 1963, Julien and Hartley 1986, Chang 1994, Coussot 1994) or for turbulent flow (Dressler 1949, Benney 1966, Brock 1969, 1970, Ponce and Simon 1977, Needham and Merkin 1984, Hwang and Chang 1987, Kranenburg 1992, Balmforth and Mandre 2004). The convective nature of the roll wave instability has been analyzed by Di Cristo and Vacca (2005) and Di Cristo et al. (2008) for constant Chézy parameterization, using the concepts of spatial stability: an initial impulse perturbation of the normal flow will thus grow while being advected downstream, e.g. Briggs (1964) or Huerre and Monkewitz (1990).

Herein, these linear analyses are extended to a large class of drag parameterization. It is demonstrated that the convective nature of the roll wave instability does not depend on the choice of drag parameterization. A quantitative comparison between the spatial (response to a boundary condition) and temporal (response to an initial condition) stability analyses in a framework moving at the wave phase velocity is also performed. Analytical results and graphs are provided, which are useful for applications dealing with open channel flows. A special attention is paid to the small and large drag limits for which the roll wave instability is replaced by marginal waves.

The Saint-Venant equations are set in Section 2. The normal flow and the drag sensitivity are defined in Section 3. In Section 4, a system of units is chosen to minimize the number of dimensionless control parameters. In Section 5, the generalized dispersion relation used for the temporal and spatial stability analyses are derived with results presented in Section 6 . The convective nature of the roll wave instability is analyzed in Section 7 with a discussion on the small and large drag limits performed in Section 8. Conclusions are finally offered.

\section{Saint-Venant equations}

Consider 1D flows slopping down a tilted and flat bottom (Fig. 1). If the horizontal scales are large compared to the flow depth, the flow can be modelled by the 1D Saint-Venant equations

$$
\begin{aligned}
& \frac{\partial h}{\partial t}+\frac{\partial}{\partial x}(U h)=0, \\
& \frac{\partial U}{\partial t}+U \frac{\partial U}{\partial x}+g \cos \alpha \frac{\partial h}{\partial x}=g \sin \alpha-\frac{1}{2} C_{f}(h, U) \frac{U|U|}{h},
\end{aligned}
$$


where $h(x, t)=$ flow depth, $U(x, t)=$ vertically-averaged velocity, and $g=$ gravity. The dimensionless function $C_{f}(h, U)=$ drag coefficient that parameterizes the turbulent momentum stress at the bottom.

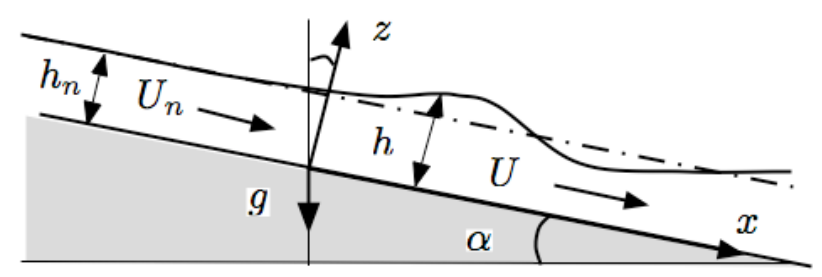

Figure 1 Free surface flow slopping down a tilted and flat bottom

The simple Chézy drag parameterization assumes that the Chézy coefficient $C_{f}$ is constant. In the Manning-Strickler parameterization, $C_{f}(h)$ is independent of $U$ and reads $C_{f}(h)=\Phi_{M S} \mathrm{Ru}^{1 / 3}$ where $\mathrm{Ru}=k_{S} /(4 h)=$ dimensionless number characterizing bottom roughness, $k_{s}=$ bottom roughness height and $\Phi_{M S}=$ dimensionless coefficient whose typical value is $\Phi_{M S}=0.05$. The Colebrook-White (1937) semi-empirical correlation is a parameterization in which $C_{f}(h, U)$ is the solution of the implicit equation $C_{f}{ }^{-1 / 2}=-4.0$ $\log _{10}\left(\mathrm{Ru} / \alpha_{f}+0.5 C_{f}^{-1 / 2} \beta_{f} / \mathrm{R}\right)$, where $\mathrm{R}=4 h U / v=$ Reynolds number, $v=$ molecular fluid viscosity and $\left(\alpha_{f}, \beta_{f}\right)=$ dimensionless coefficients of which typical values for open channel flows are $\alpha_{f}=3$ and $\beta_{f}=2.5$. These three examples are part of a large variety of parameterizations found in the literature. It is considered here that $C_{f}(h, U)$ is an arbitrary derivable and positive function.

\section{Normal flow and drag sensitivity}

If $C_{f}$ does not vanish, the homogeneous and steady model solutions, also called 'normal flow' (subscript $n)$, is $(h, U)=\left(h_{n}, U_{n}\right)$, where the constants $h_{n}$ and $U_{n}$ satisfy $2 g h_{n} \sin \alpha=C_{n} U_{n}^{2}$ with $C_{n}=C_{f}\left(h_{n}, U_{n}\right)$ for $U_{n}>0$ that will be considered from now. The two dimensionless functions

$$
\mu(h, U)=-\frac{h}{C_{f}} \frac{\partial C_{f}}{\partial h} \quad \text { and } \quad \chi(h, U)=\frac{1}{2} \frac{U}{C_{f}} \frac{\partial C_{f}}{\partial U}
$$

express the sensitivity of the drag coefficient with respect to $h$ or $U$.

The Chézy parameterization (constant $C_{f}$ ) corresponds to $\mu=\chi=0$, the Manning-Strickler parameterization $C_{f}(h)$ is such that $(\mu, \chi)=(1 / 3,0)$ and the Colebrook-White parameterization 
leads to typical values of $\mu \in[0.2,0.4]$ and $\chi \in[-0.1,0]$. The two constants $\mu=\mu\left(h_{n}, U_{n}\right)$ and $\chi_{n}=\chi\left(h_{n}, U_{n}\right)$ denote their values for the normal flow.

\section{Dimensionless controlling parameters}

The dimensionless functions $h *(x *, t)$ and $U_{*}(x * t *)$ are defined by $x=L_{0} x_{*}, t=\left(L_{0} / U_{0}\right) t *, h=h_{0} h_{*}$ and $U_{*}=U_{0} U_{*}$. With this choice of reference scales, the dimensionless Saint-Venant equations read

$$
\begin{aligned}
& \frac{\partial h_{*}}{\partial t_{*}}+\frac{\partial}{\partial x_{*}}\left(U_{*} h_{*}\right)=0, \\
& \varepsilon_{0}\left[\frac{\partial U_{*}}{\partial t_{*}}+U_{*} \frac{\partial U_{*}}{\partial x_{*}}+\frac{1}{\mathrm{~F}_{0}^{2}} \frac{\partial h_{*}}{\partial x_{*}}\right]=\frac{1}{2} C_{0}\left[1-\phi_{0}\left(h_{*}, U_{*}\right) \frac{U_{*}\left|U_{*}\right|}{h_{*}}\right],
\end{aligned}
$$

where $\varepsilon_{0}=h_{0} / L_{0}, \mathrm{~F}_{0}=U_{0} /\left(g h_{0} \cos \alpha\right)^{1 / 2}=$ Froude number, $C_{0}=C_{f}\left(h_{0}, U_{0}\right)=$ drag coefficient and $\phi_{0}\left(h_{*}, U_{*}\right)=C_{f}\left(h_{0} h_{*}, U_{0} U_{*}\right) / C_{f}\left(h_{0}, U_{0}\right)=$ renormalized drag function.

Since shallow water is considered, $\varepsilon_{0} \ll 1$. In the following, restriction to the case $F_{0}=O(1)$ is made. Three cases are relevant: small drag limit $\varepsilon_{0} \ll C_{0}$, large drag limit $C_{0} \ll \varepsilon_{0}$ and the general case. In the latter, $\varepsilon_{0}=C_{0}$ can be chosen, which reads $L_{0}=h_{0} / C_{f}\left(h_{0}, U_{0}\right)$, so that $\mathrm{F}_{0}$ and $\phi_{0}\left(h_{*}, U_{*}\right)$ are the only control parameters of Eqs. (4) and (5). The $C_{f} \rightarrow 0$ and $C_{f} \rightarrow \infty$ drag limits are then recovered by respectively looking at the small or large $x$ and $t$ scales. This point of view is adopted in the following.

\section{Temporal and spatial stability analyses}

Choosing $h_{0}=h_{n}, U_{0}=U_{n}$ and $L_{0}=h_{n} / C_{n}$, the dimensionless 1D Saint-Venant equations read

$$
\begin{aligned}
& \frac{\partial h}{\partial t}+\frac{\partial}{\partial x}(U h)=0, \\
& \frac{\partial U}{\partial t}+U \frac{\partial U}{\partial x}+\frac{1}{\mathrm{~F}_{n}^{2}} \frac{\partial h}{\partial x}=\frac{1}{2}\left[1-\phi_{n}(h, U) \frac{U|U|}{h}\right],
\end{aligned}
$$

where all subscripts $*$ were dropped for reading simplicity. The normal Froude number $\mathrm{F}_{n}=$ $U_{n} /\left(g h_{n} \cos \alpha\right)^{1 / 2}$ and the normal drag function $\phi_{n}(h, U)=C_{f}(h, U) / C_{n}$ are the only dimensionless parameters controlling this set of equations. 
The small dimensionless perturbations $h=1+\tilde{h}$ and $U=1+\tilde{U}$ of dimensionless normal flow $(h, U)=(1,1)$ obey the linear equations

$$
\begin{aligned}
& \frac{\partial \tilde{h}}{\partial t}+\frac{\partial \tilde{h}}{\partial x}+\frac{\partial \tilde{U}}{\partial x}=0, \\
& \frac{\partial \tilde{U}}{\partial t}+\frac{\partial \tilde{U}}{\partial x}+\frac{1}{\mathrm{~F}_{n}^{2}} \frac{\partial \tilde{h}}{\partial x}=\frac{1+\mu_{n}}{2} \tilde{h}-\left(1+\chi_{n}\right) \tilde{U},
\end{aligned}
$$

where $\mu_{n}=\mu\left(h_{n}, U_{n}\right)$ and $\chi_{n}=\chi\left(h_{n}, U_{n}\right)$ are defined by Eq. (3). Their complex solutions are of the form $(\tilde{h}, \tilde{U})=\left(\tilde{h}_{m}, \tilde{U}_{m}\right) \cdot \exp (\gamma x+\sigma t) \cdot \exp (\mathrm{i} k x-\mathrm{i} \omega t)$. By setting $\lambda=\sigma-\mathrm{i} \omega$ and $\kappa=\gamma+\mathrm{i} k$ the generalized dispersion relation reads

$$
\lambda^{2}+2 \lambda \kappa+\left(1-\frac{1}{\mathrm{~F}_{n}^{2}}\right) \kappa^{2}+\left(1+\chi_{n}\right) \lambda+\frac{1}{2}\left(3+\mu_{n}+2 \chi_{n}\right) \kappa=0
$$

The temporal stability analysis is obtained by setting $\gamma=0$ and looking for two branches of roots $\lambda_{ \pm}(k)=\sigma_{ \pm}(k)-\mathrm{i} \omega_{ \pm}(k)$ of the second order equation upon $\lambda$ obtained for any given $k$. The spatial stability analysis is obtained by setting $\omega$ in Eq. (10) and looking for two branches of roots $\kappa_{ \pm}(\omega)=\gamma_{ \pm}(\omega)+\mathrm{i} k_{ \pm}(\omega)$ of the second order equation upon $\lambda$ obtained for any given $\omega$. These generalized dispersion relations are analytically derived in Appendix 1.

\section{Roll-wave instability}

Figure 2 shows that the amplification rates and the phase velocity of the temporal and spatial corresponding modes are almost similar for the 'upper branch of modes', issued from the marginal $k=0$ mode, and significantly different for the 'lower branch', issued from the damped $k=0$ mode. One can indeed see that the temporal and spatial stability analyzes on the upper branch of modes are such that $\omega(k)$ and $k(\omega)$ are close to be inverse functions of each other, and that $\sigma(k)$ and $\gamma[\omega(k)] \omega(k) / k$, which is the apparent growth rate in the frame moving at phase velocity, are closely related. On the lower branch of modes, the comparison between $[\mathrm{k}, \sigma(k)]$ and $\left[\omega, \varepsilon \gamma_{-}(\omega) \omega / k_{-}(\omega)\right]$ must satisfy $\varepsilon=-1$ in the sub-critical case $\mathrm{F}_{n} \leq 1$, whereas $\varepsilon=1$ for the super-critical case $F_{n} \geq 1$. This is due to the fact that the phase velocity $\omega / k_{-}(\omega)$ of this mode changes signs at criticality. 


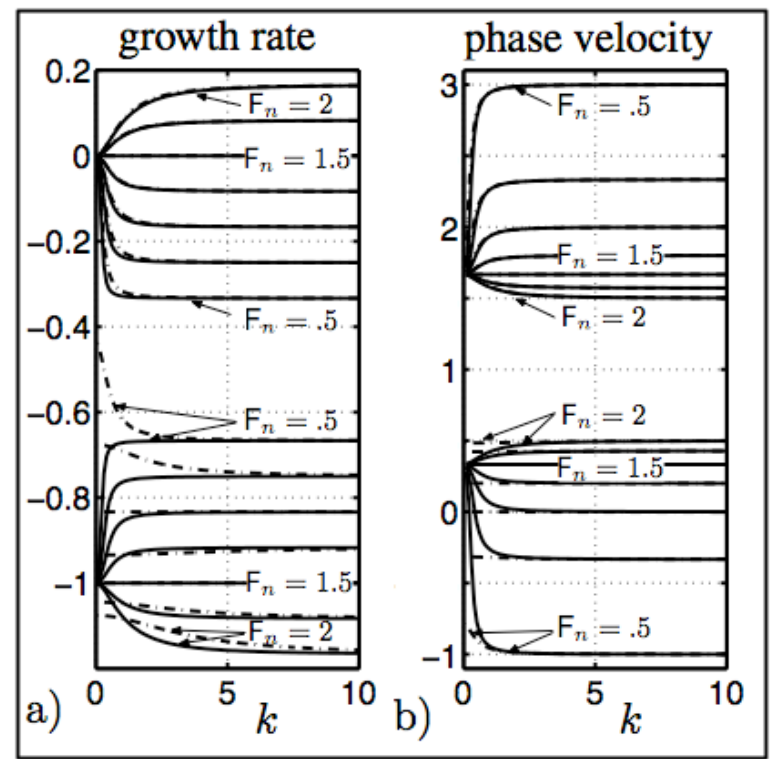

Figure 2 Generalized dispersion relations for $F_{n} \in[.5,2], \mu_{n}=1 / 3$ and $\chi_{n}=0$. Temporal stability (plain) versus spatial stability (dot-dashed); (a) Above $\left[k, \sigma_{+}(k)\right]$ versus $\left[k_{+}(\omega), \gamma_{+}(\omega) \omega / k_{+}(\omega)\right]$; below $\left[k, \sigma_{-}(k)\right]$ versus $\left[k_{-}(\omega), \varepsilon \gamma_{-}(\omega) \omega / k_{-}(\omega)\right]$ with $\varepsilon=-1$ if $F_{n} \leq 1$ and $\varepsilon=1$ if $\mathrm{F}_{n} \geq 1$; (b) above $\left[k, \omega_{+}(k) / k\right]$ versus $\left[k_{+}(\omega), \omega / k_{+}(\omega)\right]$; below $\left[k, \omega_{-}(k) / k\right]$ versus

$$
\left[k_{-}(\omega), \omega / k_{-}(\omega)\right]
$$

The critical Froude number $F_{c}$ for the onset of both temporal and spatial instabilities is obtained by setting $\lambda=-\mathrm{i} \omega$ and $\kappa=\mathrm{i} k$ in Eq. (10). This leads to

$$
\omega=\xi_{n} k \quad \text { and } \quad \mathrm{F}_{c}\left(\mu_{n}, \chi_{n}\right)=\frac{2\left(1+\chi_{n}\right)}{1+\mu_{n}}
$$

where $\xi_{n}=\left(3+\mu_{n}+2 \chi_{n}\right) /\left[2\left(1+\chi_{n}\right)\right]=1+1 / F_{c}$. For the values $\left(\mu_{n}, \chi_{n}\right)=(1 / 3,0)$ associated with the Manning-Strickler parameterization, the modes of the upper branch are marginal and nondispersive for $F_{n}=F_{c}(1 / 3,0)=3 / 2$ with a phase velocity $\xi_{n}=5 / 3$ (Fig. 2). The influence of $\mu_{n}$ on the generalized dispersion relations is shown in Fig. 3 where $F_{n}=3 / 2, \mu_{n} \in[0,1 / 2]$ and $\chi_{n}=0$. 


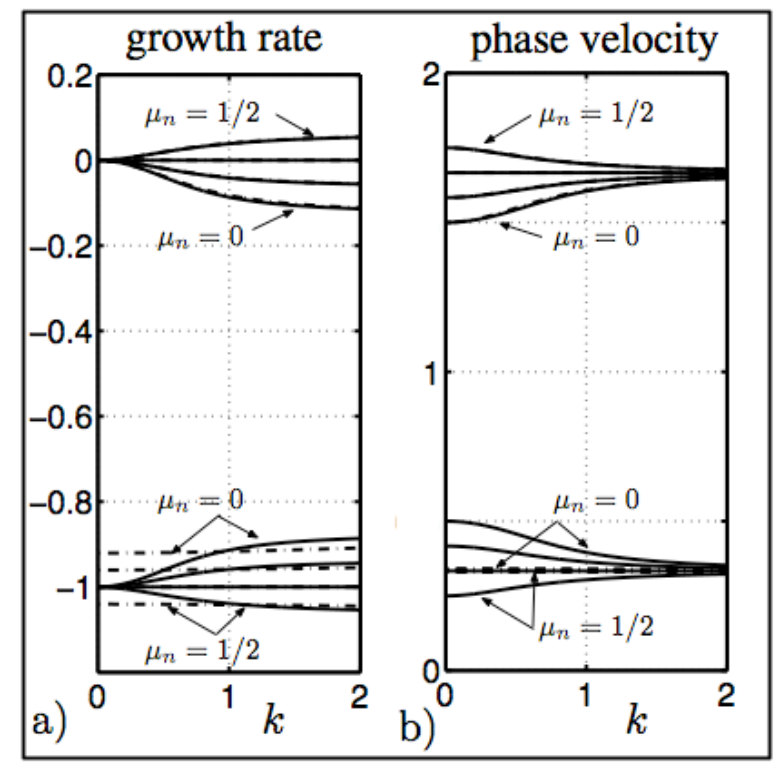

Figure 3 Generalized dispersion relations for $F_{n}=1.5, \mu_{n} \in\{0,1 / 6,1 / 3,1 / 2\}$ and $\chi_{n}=0$. Same curves as in Fig. 2

\section{Convective nature of roll wave instability}

To conclude the stability analysis of the linear Eqs. (8) and (9), it has to be determined whether the roll wave instability is of convective or absolute type. In a framework moving with the arbitrary velocity $c$, the new generalized dispersion relations $\Lambda(k)=\Sigma(k)-\mathrm{i} \Omega(k)$ are obtained by replacing $\lambda$ by $\Lambda-c \kappa$ in Eq. (10), which leads to

$$
\left[(1-c)^{2}-\frac{1}{\mathrm{~F}_{n}^{2}}\right] \kappa^{2}+\left[\Lambda^{2}(1-c)+\frac{1}{2}\left(3+\mu_{n}+2 \chi_{n}\right)-c\left(1+\chi_{n}\right)\right] \kappa+\Lambda^{2}+\left(1+\chi_{n}\right) \Lambda=0,
$$

and performing a temporal stability analysis $(\kappa=\mathrm{i} k)$ with this new equation. The response of the linear system to an initial impulse $(\tilde{h}, \tilde{U})=\left(h_{m}, U_{m}\right) \cdot \delta(x) \cdot \delta(t)$ depends on the behavior at large $t$ of the integrals $\int_{R} e^{\Lambda(k) t} \mathrm{~d} k$ for all branches of solutions $\Lambda_{ \pm}(k)$. The steepest descent method shows that this behavior depends of the vicinity of the saddle point $\kappa_{a} \in \boldsymbol{C}$ such that $\mathrm{d} \Lambda / \mathrm{d} \kappa\left(\kappa_{a}\right)=0$. If one of the real parts of the solutions $\Lambda_{a}=\Lambda\left(\kappa_{a}\right)$ of Eq. (12) is positive for $\kappa=\kappa_{a}$, the flow depth increases with time in the moving framework. The saddle nodes $\left(\kappa_{a}, \Lambda_{a}\right)$ are found if the second order polynomial in $\kappa$ of Eq. (12) has a double root, that is if $\Lambda_{a}$ is, after some algebra, solution of the equation

$$
\frac{1}{\mathrm{~F}_{n}^{2}} \Lambda^{2}+\left(1+\chi_{n}\right)\left[\frac{1}{\mathrm{~F}_{n}^{2}}+\frac{1}{\mathrm{~F}_{c}}(1-c)\right] \Lambda+\left(\frac{1+\chi_{n}}{2}\right)^{2}\left(\frac{1}{\mathrm{~F}_{c}}+1-c\right)^{2}=0 .
$$


The real part of their solutions $\Lambda_{a}$ is negative if $c \leq 1+\mathrm{F}_{c} / \mathrm{F}_{n}{ }^{2}$ and one of them vanishes, with no change of sign of the real part, for $c=1+1 / F_{c}$. Both solutions are real if $F_{n} \geq F_{c}$ and $c \notin] 1-1 / \mathrm{F}_{n}, 1+1 / \mathrm{F}_{n}\left[\right.$ or if $\mathrm{F}_{n} \leq \mathrm{F}_{c}$ and $c \in\left[1-1 / \mathrm{F}_{n}, 1+1 / \mathrm{F}_{n}\right]$. Since the real part of the two solutions $\Lambda_{a}$ are real and strictly negative for $c=0$, the instability is of convective type in the fixed framework: the growth of an initial perturbation, if any, is wiped out from any fixed point.

\section{Small and large drag limits}

The analysis of these generalized dispersion relations is completed in Appendix 1 by presenting their asymptotic expansions for large and small $(k, \omega)$, corresponding to the small and large drag limits, respectively, as discussed above. Non-dispersive and marginal modes are obtained in both cases with two families $\omega_{ \pm}(k) \approx\left(1 \pm 1 / \mathrm{F}_{\mathrm{n}}\right) k$ for small drag and only one mode $\omega_{+}(k) \approx \xi_{n} k=\left(1+1 / \mathrm{F}_{\mathrm{c}}\right) k$ for large drag.

Taking small $C_{n}$ for the small drag limit is equivalent to a backward zoom with $k, \omega, \gamma$ and $\sigma \rightarrow \infty$ (Fig. 2). At this large scale, both modes are almost marginal since $\gamma$ and $\sigma$ are of order 1 . They are also almost non-dispersive since their phase velocities converge to constant limits.

Taking large $C_{n}$ for the large drag limit is equivalent to a forward zoom with $k, \omega, \gamma$ and $\sigma$ $\rightarrow 0$ (Fig. 2). Then, only one almost marginal and non-dispersive mode is present. Note the large literature on the 'relaxation limit' for hyperbolic systems (Liu and Natalini 2001, Jin and Katsoulakis 2000, Katsoulakis and Jin 2000, Dafermos 2000, Liu 1987, or Chen et al. 1994), generalizing the present linear analysis to a nonlinear equation. For the constant $C_{f}$ case $\left(\lambda_{n}=\right.$ 0 and $\mu_{n}=0$ ), the asymptotic form of the Saint-Venant equations reads

$$
\frac{\partial h}{\partial t}+\frac{\partial}{\partial x}\left(h^{3 / 2}\right)=\left(\frac{1}{\mathrm{~F}_{n}^{2}}-\frac{1}{4}\right) \frac{\partial}{\partial x}\left(h^{3 / 2} \frac{\partial h}{\partial x}\right),
$$

and is well-posed as an initial value problem in the stable case $\mathrm{F}_{n} \leq \mathrm{F}_{c}=2$.

\section{Conclusions}

The detailed stability properties of the normal flow of the 1D Saint-Venant equations forced by gravity on a titled bottom were computed for a wide class of drag parameterizations. It was demonstrated that this analysis only depends on the normal Froude number and two dimensionless parameters expressing the sensitivity of the drag coefficient with the normal 
flow depth and normal velocity. The critical Froude numbers at the onset of the roll wave instability was computed indicating that this instability was of convective type. The regions of the control parameter where temporal and spatial stabilities are not close to each other were also identified. All these observations are useful to compare between real flows and their modeling with the Saint-Venant equations.

\section{Appendix 1}

The analytical solutions of Eq. (10) is

$$
\lambda^{2}+2 \lambda \kappa+\left(1-\frac{1}{\mathrm{~F}_{n}^{2}}\right) \kappa^{2}+\left(1+\chi_{n}\right) \lambda+\frac{1}{2}\left(3+\mu_{n}+2 \chi_{n}\right) \kappa=0
$$

for both temporal $\kappa=\mathrm{i} k$ and spatial stability $\lambda=-\mathrm{i} \omega$ analyses. For the temporal stability analysis, set $\kappa=\mathrm{i} k$ and look at the complex solutions $\lambda=\sigma-\mathrm{i} \omega$ of

$$
\lambda^{2}+\left(1+\chi_{n}+2 \text { i } k\right) \lambda+\left(\frac{1}{\mathrm{~F}_{n}^{2}}-1\right) k^{2}+\frac{1}{2}\left(3+\mu_{n}+2 \chi_{n}\right) \text { i } k=0 .
$$

The two solutions $\lambda_{ \pm}(k)=\sigma_{ \pm}(k)+\mathrm{i} \omega_{ \pm}(k)$ are

$$
\lambda_{ \pm}(k)=-\frac{1+\chi_{n}}{2}-\mathrm{i} k \pm \sqrt{\left(\frac{1+\chi_{n}}{2}\right)^{2}-\frac{1+\mu_{n}}{2} \mathrm{i} k-\frac{1}{\mathrm{~F}_{n}^{2}} k^{2}} .
$$

The asymptotic expansions for small $k$ read

$$
\begin{array}{ll}
\sigma_{+}(k) \approx \frac{k^{2}}{1+\chi_{n}} \frac{\mathrm{F}_{n}^{2}-\mathrm{F}_{c}^{2}}{\mathrm{~F}_{n}^{2} \mathrm{~F}_{c}^{2}}+\mathrm{O}\left(k^{3}\right), & \omega_{+}(k) \approx\left(1+\frac{1}{\mathrm{~F}_{c}}\right) k+\mathrm{O}\left(k^{2}\right), \\
\sigma_{-}(k) \approx-\left(1+\chi_{n}\right)+\mathrm{O}\left(k^{2}\right), & \omega_{-}(k) \approx\left(1-\frac{1}{\mathrm{~F}_{c}}\right) k+\mathrm{O}\left(k^{2}\right),
\end{array}
$$

with $\mathrm{F}_{c}\left(\mu_{n}, \chi_{n}\right)=2\left(1+\chi_{n}\right) /\left(1+\mu_{n}\right)$. The asymptotic expansions for large $k$ read

$$
\begin{array}{ll}
\sigma_{+}(k) \approx \frac{1+\mu_{n}}{4}\left(\mathrm{~F}_{n}-\mathrm{F}_{c}\right)+\mathrm{O}\left(\frac{1}{k}\right), & \omega_{+}(k) \approx\left(1+\frac{1}{\mathrm{~F}_{n}}\right) k+\mathrm{O}(1), \\
\sigma_{-}(k) \approx \frac{1+\mu_{n}}{4}\left(-\mathrm{F}_{n}-\mathrm{F}_{c}\right)+\mathrm{O}\left(\frac{1}{k}\right), & \omega_{-}(k) \approx\left(1-\frac{1}{\mathrm{~F}_{n}}\right) k+\mathrm{O}(1) .
\end{array}
$$

For the spatial stability analysis, set $\lambda=-\mathrm{i} \omega$ and look at the complex solutions $\kappa=\gamma+\mathrm{i} k$ of

$$
\kappa^{2}\left(1-\frac{1}{\mathrm{~F}_{n}^{2}}\right)+\kappa\left[\frac{1}{2}\left(3+\mu_{n}+2 \chi_{n}\right)-2 \mathrm{i} \omega\right]-\omega^{2}-\left(1+\chi_{n}\right) \mathrm{i} \omega=0
$$

If $1 / \mathrm{F}_{n}{ }^{2} \neq 1$, the two solutions $\kappa_{ \pm}(\omega)=\gamma_{ \pm}(\omega)+\mathrm{i} k_{ \pm}(\omega)$ are

$$
\kappa_{ \pm}(\omega)=\frac{1}{1-\frac{1}{\mathrm{~F}_{n}^{2}}}\left[-\frac{3+\mu_{n}+2 \chi_{n}}{4}+\mathrm{i} \omega \pm \sqrt{\left(\frac{3+\mu_{n}+2 \chi_{n}}{4}\right)^{2}-\mathrm{i} \omega\left(\frac{1+\mu_{n}}{2}+\frac{1+\chi_{n}}{\mathrm{~F}_{n}^{2}}\right)-\frac{\omega^{2}}{\mathrm{~F}_{n}^{2}}}\right]
$$


For $1 / \mathrm{F}_{n}{ }^{2}=1$, a continuity argument indicates that the unique solution of Eq. $(23)$ is $\kappa_{+}(\omega)=$ $\gamma_{+}(\omega)+\mathrm{i} k_{+}(\omega)$ with

$$
\kappa_{+}(\omega)=\frac{\omega^{2}+\left(1+\chi_{n}\right) \mathrm{i} \omega}{\frac{1}{2}\left(3+\mu_{n}+2 \chi_{n}\right)-2 \mathrm{i} \omega},
$$

while the curve $\left[\omega, k_{-}(\omega)\right]$ becomes the straight $\omega=0$ and the curve $\left[\omega, \gamma_{-}(\omega) \omega / k_{-}(\omega)\right]$ the straight $\gamma \omega / k=-\left(3+\mu_{n}+2 \chi_{n}\right) / 4$.

The asymptotic expansions for small $\omega$ read

$$
\begin{gathered}
k_{+}(\omega) \approx\left(1+\frac{1}{\mathrm{~F}_{c}}\right)^{-1} \omega+\mathrm{O}\left(\omega^{2}\right), \quad k_{-}(\omega) \approx \frac{\mathrm{F}_{n}^{2}\left(1+\frac{2}{\mathrm{~F}_{c}}\right)+1}{\left(\mathrm{~F}_{n}^{2}-1\right)\left(1+\frac{1}{\mathrm{~F}_{c}}\right)} \omega+\mathrm{O}\left(\omega^{2}\right), \\
\frac{\gamma_{+}(\omega) \omega}{k_{+}(\omega)} \approx \sigma_{+}\left[k_{+}(\omega)\right]+\mathrm{O}\left(\omega^{3}\right), \quad \frac{\gamma_{-}(\omega) \omega}{k_{-}(\omega)} \approx \frac{1+\mu_{n}}{2} \frac{\mathrm{F}_{n}^{2} \mathrm{~F}_{c}\left(1+\frac{1}{\mathrm{~F}_{c}}\right)}{\mathrm{F}_{n}^{2}\left(1+\frac{2}{\mathrm{~F}_{c}}\right)+1} \omega+\mathrm{O}(\omega) .
\end{gathered}
$$

\section{Notations}

$C_{f}=$ Chézy coefficient

$\mathrm{F}_{c}=$ Critical Froude number for roll wave instability

$g=$ Gravity acceleration

$h=$ Flow depth

$k=$ Wave number

$k_{s}=$ Roughness height

$L_{0}=$ Horizontal unit length

$\mathrm{R}=$ Reynolds number

$\mathrm{Ru}=$ Roughness number

$t=$ Time

$U=$ Mean streamwise velocity

$x=$ Streamwise coordinate

$z=$ Coordinate normal to bottom

$\alpha=$ Angle of inclined plane with horizontal

$\alpha_{f}=$ Coefficient of Colebrook-White formula

$\beta_{f}=$ Coefficient of Colebrook-White formula

$\delta=$ Dirac function 
$\varepsilon_{0}=$ Dimensionless number $h_{0} / L_{0}$

$\phi=$ Notation for $C_{f} / C_{n}$ or $C_{f} / C_{0}$

$\Phi_{M S}=$ Coefficient of Manning-Strickler parameterization

$\chi(\omega)=$ Spatial wave growth rate

$\kappa=$ Notation for $\gamma+\mathrm{i} k$

$\lambda=$ Notation for $\sigma-\mathrm{i} \omega$

$\Lambda(k)=$ Generalized dispersion relation

$\mu=$ Dimensionless derivative of $C_{f}$ with $h$

$v=$ Molecular viscosity

$\chi=$ Dimensionless derivative of $C_{f}$ with $U$

$\sigma(k)=$ Temporal wave growth rate

$\xi=$ Constant expressed with $\mu$ and $\chi$

$\omega=$ Temporal wave pulsation

$\sim=$ Notation for perturbations

\section{Subscripts}

${ }_{a}=$ Index for values at saddle point

${ }_{m}=$ Index for complex amplitudes

${ }_{n}=$ Index for normal flow fields

* = Index for non-dimensional fields

${ }_{+}=$Index for upper branch of modes

_ = Index for lower branch of modes

\section{References}

Akan, A.O. (2006). Open channel hydraulics. Elsevier, Oxford UK.

Balmforth, N.J., Mandre, S. (2004). Dynamics of roll waves. J. Fluid Mech. 514, 1-33.

Benjamin, T.B. (1957). Wave formation in laminar flow down an inclined plane. J. Fluid Mech. 2, 554-574.

Benney, D.J. (1966). Long waves on liquid films. J. Math. Phys. 54, 150-155.

Briggs, R.J. (1964). Electron-steam interaction with plasmas. MIT Press, Cambridge MA.

Brock, R.R. (1969). Development of roll-waves trains in open channel. J. Hydraulics Div. ASCE 95(HY4), 1401-1427.

Brock, R.R. (1970). Periodic permanent roll waves. J. Hydraulics Div. ASCE 96(HY12), 2565-2580. 
Chang, H.-C. (1994). Wave evolution on a falling film. Ann. Rev. Fluid Mech. 26, 103-136.

Chen, G.-Q., Levermore, C.D., Liu, T.-P. (1994). Hyperbolic conservation laws with stiff relaxation terms and entropy. Comm. Pure and Applied Math. 47(6), 787-830.

Chow, V.T. (1959). Open channel hydraulics. McGraw-Hill, New York.

Colebrook, C.F., White, C.M. (1937). Experiments with fluid friction in roughened pipes. Proc. R. Soc. London A, 161, 367-381.

Cornish, V. (1910). Ocean waves and kindred geophysical phenomena. Cambridge University Press, Cambridge UK.

Coussot, P. (1994). Steady, laminar, flow of concentrated mud suspensions in open channel. J. Hydraulic Res. 32(4), 535-560.

Dafermos, C.M. (2000). Hyperbolic conservation laws in continuum physics. Springer, Berlin.

Di Cristo, C., Iervolino, M., Vacca, A., Zanuttigh, B. (2008). Minimum channel length for roll-wave generation. J. Hydraulic Res. 46(1), 73-79.

Di Cristo, C., Vacca, V. (2005). On the convective nature of roll waves instability. J. Appl. Math. 3, 259-271.

Dressler, R.F. (1949). Mathematical solution of the problem of roll waves in inclined channel flows. Comm. Pure Appl. Math. 2(2-3), 149-194.

Huerre, P., Monkewitz, P.A. (1990). Local and global instabilities in spatially developing flows. Ann. Rev. Fluid Mech. 22, 473-537.

Hwang, S.-H., Chang, H.-C. (1987). Turbulent and inertial roll waves in inclined film flow. Phys. Fluids 30(5), 1259-1268.

Jeffreys, H. (1925). The flow of water in an inclined channel of rectangular bottom. Phil. Mag. Ser. 6 49, 793-807.

Jin, S., Katsoulakis, M.A. (2000). Hyperbolic systems with supercharacteristic relaxations and roll waves. SIAM J. Appl. Math. 61(1), 273-292.

Julien, P.Y., Hartley, D.M. (1986). Formation of roll waves in laminar sheet flow. J. Hydraulic Res. 24(1), 5-17.

Kapitza, P.L., Kapitza, S.P. (1949). Wave flow of thin viscous liquid films. Zh. Exper. i Teor. Fiz. 19, 105-120.

Katsoulakis, M.A., Jin, S. (2000). Hyperbolic systems with super-characteristic relaxations and roll waves. SIAM J. Appl. Math. 61(1), 273-292.

Kranenburg, C. (1992). On the evolution of roll waves. J. Fluid Mech. 245, 249-261.

Liu, H., Natalini, R. (2001). Long-time diffusive behavior of solutions to a hyperbolic relaxation system. Asymp. Anal. 25(1), 21-38. 
Liu, T.-P. (1987). Hyperbolic conservation laws with relaxation. Comm. Math. Physics 108(1), 153-175.

Needham, D.J., Merkin, J.H. (1984). On roll waves down an open inclined channel. Proc. R. Soc. London A 394(1807), 259-278.

Ponce, V.M., Simons, D.B. (1977). Shallow water propagation in open channel flow. J. Hydraulics Div. ASCE 103(HY12), 1461-1476.

Yih, C.-S. (1963). Stability of liquid flow down an inclined plane. Phys. Fluids 6, 321-333. 